\title{
Riesgo cardiovascular en una población adolescente de Timbio, Colombia
}

\section{Cardiovascular risk in a teenage population in Timbio, Colombia}

\author{
Clara-I Torres-A ${ }^{1}$; Diego Illera ${ }^{1}$; Darly-E Acevedo-B ${ }^{1}$; Mayra Cadena-M ${ }^{1}$; Luis-C Meneses-M ${ }^{1}$; \\ Paola-A Ordoñez-H ${ }^{1}$; Lizeth-N Pantoja-A ${ }^{1}$; Marcela Pastás-C ${ }^{1}$
}

Forma de citar: Torres CI, Illera D, Acevedo DE, Cadena M, Meneses LC, Ordoñez PA, Pantoja LN, Pastás M. Riesgo cardiovascular en una población adolescente de Timbío, Colombia. Rev Univ Ind Santander Salud. 2018; 50(1): 59-66. doi: http://dx.doi.org/10.18273/revsal.v50n1-2018006 @) (1)

\section{Resumen}

Introducción: Las enfermedades cardiovasculares se encuentran entre las primeras causas de muerte a nivel mundial y la evidencia científica sugiere que tienen un origen en edades tempranas, por lo que disminuir desde la adolescencia los riesgos que contribuyen a su aparición es indispensable. Objetivo: Determinar riesgo cardiovascular en la población adolescente de una institución educativa de Timbío, Cauca, 2015 - 2016. Metodología: Estudio cuantitativo, descriptivo, observacional y de corte transversal. Muestra: 100 estudiantes (47 mujeres, 53 hombres), de 10 a 19 años de edad. Se evaluaron variables sociodemográficas, antropométricas, fisiológicas, bioquímicas (glicemia), antecedentes personales, familiares, estilos de vida y uso de anticonceptivos orales. Resultados: Se encontró una mayor prevalencia de riesgo cardiovascular en el grupo etario adolescente (15-18 años) con 84.6\%; el género femenino presentó más posibilidad de tener riesgo cardiovascular (OR: 2.8, IC 95\% 1.17 - 6.87); el $7 \%$ afirmó consumir tabaco regularmente y el $12 \%$ son fumadores pasivos. Se obtuvo que el $26 \%$ ingiere bebidas alcohólicas, con una mayor prevalencia entre las edades de 15 a 18 años (OR: 19.4, IC 95\% 2.45-48.11); en cuanto a la actividad física el $79 \%$ la practicaba de forma extracurricular. Presentar hábitos alimenticios no saludables genera mayor riesgo cardiovascular (OR: 5.57, IC 95\% 1.95 - 15.90). El 67\% ( $\mathrm{n}=67)$ tiene riesgo cardiovascular. Conclusiones: El género femenino, el grupo adolescente (15-18 años), el consumo de bebidas alcohólicas y hábitos alimenticios no saludables, se asocian con una mayor posibilidad de tener riesgo cardiovascular. Se encontró en un gran porcentaje de la población de estudio riesgo cardiovascular.

Palabras clave: Factores de riesgo; Enfermedades cardiovasculares; Estilo de vida; Niño; Adolescente.

\begin{abstract}
Introduction: Cardiovascular diseases are among the leading causes of death worldwide and scientific evidence suggests that they have an origin at early ages, so that diminishing from adolescence the risks that contribute to its emergence is essential. Objective: To determine cardiovascular risk in the adolescent population of a high school of Timbío, Cauca, 2015 - 2016. Methodology: Quantitative, descriptive, observational and cross-sectional study. Sample: 100 students (47 women, 53 men), from 10 to 19 years old. Sociodemographic, anthropometric, physiological, biochemical variables (glycemia), personal history, family history, lifestyles and use of oral contraceptives were evaluated. Results: A higher prevalence of cardiovascular risk was found in the adolescent age group (15-18 years)

1. Universidad del Cauca. Popayán, Colombia.

Correspondencia: Lizeth Nataly Pantoja Álvarez. Portal de la Colina, Manzana F, casa 66. Popayán, Colombia. Correo electrónico: natalypantoja910@gmail.com. Teléfono:+573175626503.
\end{abstract}


with $84.6 \%$; female gender was more likely to have cardiovascular risk (OR: $2.8,95 \%$ CI 1.17 - 6.87); $7 \%$ said they use tobacco regularly and $12 \%$ are passive smokers. It was obtained that $26 \%$ ingested alcoholic beverages, with a higher prevalence between the ages of 15 to 18 years (OR: 19.4, 95\% CI 2.45-48.11); In terms of physical activity, $79 \%$ practiced it extracurricularly. Presenting unhealthy eating habits generates greater cardiovascular risk (OR: 5.57, 95\% CI 1.95 - 15.90). 67\% $(\mathrm{n}=67)$ have cardiovascular risk. Conclusions: The female gender, the adolescent group (15-18 years), drink alcoholic beverages and unhealthy eating habits, are associated with a greater possibility of having cardiovascular risk. It was found in a large percentage of the study population cardiovascular risk.

Keywords: Risk factors; Cardiovascular diseases; Life style; Child; Adolescent.

\section{Introducción}

Las enfermedades cardiovasculares (ECV) son un grupo de desórdenes del corazón y de los vasos sanguíneos ${ }^{1}$, las cuales según la Organización Mundial de la Salud (OMS) son las responsables de 17 millones de muertes en el mundo, y se estima que para el año 2020, las muertes por estas enfermedades aumentaran de $15 \%$ a $20 \%$, En Colombia, en los últimos 30 años las enfermedades cardiovasculares (ECV) han ocupado los primeros cinco puestos en la lista de las principales causas de mortalidad de la población ${ }^{4}$.

La pérdida de productividad a causa de las patologías cardiovasculares es sustancial, ante la cantidad de personas afectadas por estas enfermedades que mueren de manera prematura por ellas ${ }^{5,6}$, acortan los años de vida productivos y deterioran la calidad de vida de las personas que las padecen, requiriendo medicamentos de por vida, cirugías complejas y hospitalizaciones prolongadas. La diabetes tipo 2 por ejemplo, se considera una pandemia de alto costo, tanto social, como económico, que por su cronicidad afecta la calidad de $v^{2} i^{7}$, empeorando las condiciones de pobreza, afectando el desarrollo económico y el bienestar de cualquier nación; no sólo tienen una alta carga para las personas, las familias y las comunidades, sino que también obstaculizan el crecimiento socioeconómico, especialmente de las personas y de los países de menores ingresos ${ }^{8}$. Sin embargo, existe una importante evidencia científica, que orienta las estrategias de prevención, detección y control de estas enfermedades, a través de respuestas costo - efectivas ${ }^{9}$ que eviten el colapso de los sistemas de salud, ya que los recursos que se destinan al tratamiento de estas enfermedades pueden invertirse en programas de protección específica y detección temprana ${ }^{10}$, que permitan reducir los gastos sanitarios, pero principalmente aumentar la esperanza de vida actual de las comunidades y mejorar la calidad de vida de quienes las padecen o están en riesgo, disminuyendo la posibilidad de someterse a tratamientos e intervenciones para curar o controlar estas enfermedades ${ }^{11}$.
Según la Organización Mundial de la Salud, los principales factores de riesgo cardiovascular que pueden ser prevenidos oportunamente son: hipertensión arterial (HTA), dislipidemias, tabaquismo, sedentarismo, obesidad, dieta poco saludable y diabetes mellitus $^{1,12}$, las cuales al ser intervenidas desde edades tempranas, hacen posible que el éxito de la prevención y el tratamiento de estas enfermedades puedan reducir su incidencia en la adultez ${ }^{13,14}$. Esto hace imperante la motivación de los agentes de salud y los gobiernos por la detección temprana de dichos factores de riesgo en población joven, pues es la mejor manera de implementar estrategias de intervención, oportunas y acertadas, teniendo un conocimiento de dichos factores. Por lo anteriormente descrito, este trabajo pretende contribuir a dar respuesta a la necesidad de investigar factores de riesgo cardiovascular en adolescentes de una institución educativa de la región y, de acuerdo a los hallazgos, hacer las recomendaciones a la institución, entorno a la promoción de hábitos saludables y prevención de enfermedades crónicas no trasmisibles, desde una temprana edad, como insumo para la creación de estrategias educativas que involucren de manera activa a los padres de familia, mitigando en alguna medida el impacto a futuro de las enfermedades crónicas no trasmisibles en nuestro medio.

\section{Metodología}

Se desarrolló un estudio cuantitativo, descriptivo, observacional de corte transversal, realizado en la institución educativa Concentración Escuela Guillermo Valencia con previa autorización del rector. La población total perteneciente a la institución estaba constituida por 467 estudiantes, una muestra calculada de 100 alumnos de bachillerato de 10 a 19 años de edad de ambos sexos. El muestreo fue probabilístico estratificado con afijación proporcional, se subdividió en grupos de acuerdo al grado escolar al que pertenecían los estudiantes; la selección de las unidades de estudio finales, se realizó por muestreo aleatorio simple. Los participantes seleccionados aceptaron su incorporación al estudio y otorgaron el consentimiento informado parental 
de forma voluntaria y el consentimiento informado del estudiante si era mayor de edad. Criterios de exclusión: adolescentes con enfermedad diagnosticada, como diabetes mellitus, cardiopatía congénita con hipertensión elevada, híper e hipotiroidismo; embarazo y sin consentimiento informado diligenciado.

La recolección de los datos se hizo mediante la técnica de encuesta dirigida, por medio de un instrumento el cual fue tomado con previa autorización de los autores del estudio: Evaluación de riesgo cardiovascular en adolescentes de segundo y tercer año de enseñanza media de establecimientos educacionales, de la ciudad de punta arenas año 2005", al que se le realizaron algunas modificaciones al simplificar el lenguaje para la comprensión por parte de los participantes de menor edad y en la especificación del estrato al cual pertenecía cada uno; posteriormente se hizo una prueba piloto en la institución, lo que permitió corregir errores en el cuestionario y en los procedimientos de aplicación del mismo. Entre los datos interrogados en el instrumento se incluyó la identificación personal y datos sociodemográficos, variables antropométricas, fisiológicas y bioquímicas y preguntas direccionadas a evaluar la presencia de factores de riesgo cardiovascular.

El instrumento fue aplicado en la población sujeto por los investigadores, se realizo toma de glicemia capilar en ayunas, con el glucómetro de la marca TRUEresult, NIPRO DIAGNOSTIC, Serie MO6528455, medición de la presión arterial con un tensiómetro análogo Marca GMD, serie 11102623, CERTIFICADO de calibración $\mathrm{N}^{\circ}$ : TA-20160110-01, Dumian Medical y finalmente para la evaluación antropométrica una báscula Marca Discover CERTIFICADO de calibración $\mathrm{N}^{\circ}$ : B-20160110-01, Dumian Medical. Esto se realizó teniendo en cuenta los parámetros establecidos para la toma de estas variables. Los datos fueron analizados mediante el programa estadístico Stata Versión 12, con medidas de tendencia central, medidas de correlación con chi cuadrado y de asociación como el OR.

Los resultados se expresan en porcentajes y se presentan en tres grupos: los resultados univariados, los bivariados, para los cuales se realiza el cruce de las variables generales con la variable dependiente: riesgo cardiovascular. Para determinar el riesgo cardiovascular se clasificó la población en: sin riesgo y con riesgo, de acuerdo al número de factores de riesgo que tuvieran, se estableció para el presente estudio, con riesgo 2 o más factores presentes y se estableció la significancia estadística p: $(<0.05)$, en relación al riesgo cardiovascular y el tercer grupo hace referencia a la asociación del riesgo cardiovascular con las variables significantes estadísticamente.

\section{Resultados}

Del total de participantes $(\mathrm{n}=100)$ con relación a las variables sociodemográficas, en la edad se encontró una mediana de 14 , un promedio de 13.9 con una DS de 2, una edad mínima de 10 años y una máxima de 18 años. Hay una mayor frecuencia de género masculino con un $53 \%(\mathrm{n}=53)$ y de los estratos socioeconómicos 1 y 2 con el $99 \%(n=99)$. En cuanto a la procedencia la mayoría pertenece a la zona rural $59 \%(\mathrm{n}=59)$.

Los valores de índice de masa corporal (IMC) mostraron que la mayoría de los estudiantes tiene un estado nutricional normal 94\% ( $\mathrm{n}=94)$. La desnutrición, sobrepeso y obesidad tuvieron una prevalencia del $2 \%$ $(\mathrm{n}=2)$ cada uno.

En las variables fisiológicas, el 95\% $(\mathrm{n}=95)$ de los encuestados presentaron una presión arterial dentro de los parámetros normales para la edad y el 93\% (n=93) una frecuencia cardiaca normal. De igual manera el $99 \%(n=99)$ de la población tiene valores de glicemia normales por medición capilar.

Respecto a los antecedentes familiares de los encuestados, el 71\% ( $\mathrm{n}=71)$ no tienen en padres y abuelos algún tipo de ECV y el 93\% (n=93) no tienen antecedentes personales.

En los estilos de vida del total de la muestra, en relación al consumo de tabaco, el 7\% $(\mathrm{n}=7)$ declaró ser fumador de los cuales el $85.7 \%(n=6)$ están entre los 15-18 años. Un 12\% $(\mathrm{n}=12)$ de los adolescentes son fumadores pasivos. También se encontró que el 26\% $(n=26)$ de los adolescentes ingieren bebidas alcohólicas, de los cuales el 84.6\% (22) estaban entre $\operatorname{los} 15$ a 18 años.

De los participantes, el 79\% $(\mathrm{n}=79)$ practicaban algún tipo de actividad física extracurricular. El 80\% $(\mathrm{n}=80)$ de los encuestados tenían malos hábitos de alimentación (consumo de grasas saturadas y comida chatarra), el 31\% ( $\mathrm{n}=31)$ ingieren todos los días frutas y el $20 \%(n=20)$ verduras; solo el $16 \%(n=16)$ no consumen grasas.

Del $47 \% \quad(n=47)$ que corresponde a la población femenina, el 14\% (7) tomaba anticonceptivos orales.

Tabla 1. 
Tabla 1. Características generales de la población de estudio.

\begin{tabular}{|c|c|c|}
\hline Variable & $\mathbf{n}$ & $(\%)$ \\
\hline \multicolumn{3}{|l|}{ VARIABLES SOCIODEMOGRÁFICAS } \\
\hline \multicolumn{3}{|l|}{ Edad } \\
\hline Pre adolecentes (9-11) & 13 & 13.0 \\
\hline Adolescente temprano (12-14) & 48 & 48.0 \\
\hline Adolescente (15-18) & 39 & 39.0 \\
\hline \multicolumn{3}{|l|}{ Género } \\
\hline Femenino & 47 & 47.0 \\
\hline Masculino & 53 & 53.0 \\
\hline \multicolumn{3}{|l|}{ Estrato } \\
\hline 1 & 75 & 75.0 \\
\hline 2 & 24 & 24.0 \\
\hline 4 & 1 & 1.0 \\
\hline \multicolumn{3}{|l|}{ Procedencia } \\
\hline Rural & 59 & 59.0 \\
\hline Urbana & 41 & 41.0 \\
\hline \multicolumn{3}{|l|}{ VARIABLES ANTROPOMÉTRICAS } \\
\hline \multicolumn{3}{|l|}{ Diagnóstico nutricional } \\
\hline Desnutrición Moderada & 2 & 2.0 \\
\hline Normal & 94 & 94.0 \\
\hline Sobrepeso & 2 & 2.0 \\
\hline Obesidad & 2 & 2.0 \\
\hline \multicolumn{3}{|l|}{ VARIABLES FISIOLÓGICAS } \\
\hline \multicolumn{3}{|l|}{ Presión Arterial } \\
\hline Normal (PAS y PAD < P90) & 95 & 95.0 \\
\hline Normal-Alta (PAS y/o PAD $\geq$ P90 pero $<$ P95) & 5 & 5.0 \\
\hline \multicolumn{3}{|l|}{ Frecuencia Cardiaca } \\
\hline \multicolumn{3}{|l|}{ Normal } \\
\hline (13 a 18 años) $60-120$ lpm & 93 & 93.0 \\
\hline Alterada & 7 & 7.0 \\
\hline \multicolumn{3}{|l|}{ VARIABLES BIOQUÍMICAS } \\
\hline \multicolumn{3}{|l|}{ Glicemia } \\
\hline Normal ( $70-110$ mg/dl)) & 99 & 99.0 \\
\hline Alterada & 1 & 1.0 \\
\hline \multicolumn{3}{|l|}{ ANTECEDENTES } \\
\hline \multicolumn{3}{|l|}{ Antecedentes personales (Diabetes, HTA, ACV) } \\
\hline No & 92 & 92.0 \\
\hline $\mathrm{Si}$ & 8 & 8.0 \\
\hline Antecedentes familiares (Diabetes, HTA, A & & \\
\hline No & 71 & 71.0 \\
\hline $\mathrm{Si}$ & 29 & 29.0 \\
\hline ESTILOS DE VIDA & & \\
\hline Consumo de tabaco & & \\
\hline No fuma & 93 & 93.0 \\
\hline Fuma ocasionalmente & 7 & 7.0 \\
\hline Fumadores pasivos & & \\
\hline No & 88 & 88.0 \\
\hline $\mathrm{Si}$ & 12 & 12.0 \\
\hline Consumo de bebidas alcohólicas & & \\
\hline No & 74 & 74.0 \\
\hline $\mathrm{Si}$ & 26 & 26.0 \\
\hline Practica actividad física & & \\
\hline $\mathrm{Si}$ & 79 & 79.0 \\
\hline No & 21 & 21.0 \\
\hline Alimentación saludable & & \\
\hline $\mathrm{Si}$ & 20 & 20.0 \\
\hline No & 80 & 80.0 \\
\hline USO DE ANTICONCEPTIVOS ORALES & & \\
\hline No & 40 & 85.1 \\
\hline $\mathrm{Si}$ & 7 & 14.9 \\
\hline RIESGO CARDIOVASCULAR & & \\
\hline Sin riesgo & 33 & 33.0 \\
\hline Con riesgo & 67 & 67.0 \\
\hline
\end{tabular}

Con relación a la edad y el riesgo cardiovascular, la mayor prevalencia se encontró en los adolescentes (1518 años) con $84.6 \%$, seguido de los preadolescentes (9-11 años) con un 61.5\%, encontrándose diferencias significativas, $(\mathrm{p}=0.010)$. Respecto al género, se encontró mayor riesgo cardiovascular, en las mujeres con un $78.7 \%(\mathrm{p}=0.019)$ y mayor riesgo cardiovascular en los consumidores de alcohol (96.2\%), con una significancia estadística, $\mathrm{p}=0.000$.

En relación a la práctica de actividad física y el riesgo cardiovascular se observó mayor prevalencia en quienes no practican ninguna, con $85.7 \%(\mathrm{p}=0.040)$. Frente a hábitos nutricionales y el riesgo cardiovascular, en aquellos con una alimentación no saludable $(75 \%)$, hay mayor riesgo $(\mathrm{p}=0.001)$. Otras variables medidas en el presente estudio no mostraron significancia estadística respecto al riesgo cardiovascular como: consumo de tabaco, diagnóstico nutricional y consumo de anticonceptivos. Tabla 2.

Tabla 2. Significancia estadística en relación al riesgo cardiovascular.

\begin{tabular}{lccccccc}
\hline \multirow{2}{*}{ Variable } & \multicolumn{2}{c}{ Sin } & \multicolumn{2}{c}{ Con } & \multirow{2}{*}{ Total } & \multirow{2}{*}{$\begin{array}{c}\text { p } \\
\text { Riesgo }\end{array}$} & \multicolumn{2}{c}{ Riesgo } & Total & & $(<0.05)$ \\
\cline { 2 - 8 } & n & $\%$ & n & $\%$ & n & $\%$ & \\
\hline
\end{tabular}

Edad (Etapas de la adolescencia)

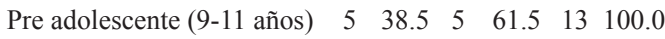

Adolecente temprano (12- $22 \quad 45.8 \quad 26 \quad 54.2 \quad 48 \quad 100.0 \quad 0.010$

14 años)

Adolescente (15-18 años) $\quad \begin{array}{lllllll}6 & 15.4 & 33 & 84.6 & 39 & 100.0\end{array}$

Género

$\begin{array}{llllllll}\text { Femenino } & 10 & 21.3 & 37 & 78.7 & 47 & 100.0 & 0.019\end{array}$

$\begin{array}{lllllll}\text { Masculino } & 23 & 43.4 & 30 & 56.6 & 53 & 100.0\end{array}$

Diagnóstico nutricional

Normal $\quad \begin{array}{llllllll}30 & 31.9 & 64 & 68.1 & 94 & 100.0 & 0.361\end{array}$

$\begin{array}{lllllll}\text { Anormal } & 3 & 50 & 3 & 50 & 6 & 100.0\end{array}$

Antecedentes personales

No

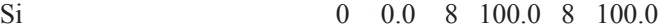

Antecedentes familiares

No $\quad \begin{array}{lllllll}33 & 46.5 & 38 & 53.5 & 71 & 100.0 & 0.000\end{array}$

$\begin{array}{lllllll}\mathrm{Si} & 0 & 0.0 & 29 & 100.0 & 29 & 100.0\end{array}$

Consumo de tabaco

$\begin{array}{llllllll}\text { No fuma } & 33 & 35.5 & 60 & 64.5 & 93 & 100.0 & 0.054\end{array}$

Fuma ocasionalmente $\quad \begin{array}{lllllll}0 & 0.0 & 7 & 100.0 & 7 & 100.0\end{array}$

Fumadores pasivos

No $\quad \begin{array}{llllllll}33 & 37.5 & 55 & 62.5 & 88 & 100.0 & 0.010\end{array}$

Si $\quad \begin{array}{lllllll}0 & 0.0 & 12 & 100.0 & 12 & 100.0\end{array}$

Consumo de bebidas alcohólicas

No $\quad \begin{array}{llllll}33 & 43.2 & 42 & 52.8 & 74 & 100.0\end{array}$

$\begin{array}{llllllll}\mathrm{Si} & 1 & 3.8 & 25 & 96.2 & 26 & 100.0 & 0.000\end{array}$

Practica actividad física

$\begin{array}{llllllll}\mathrm{Si} & 30 & 38 & 49 & 62 & 79 & 100.0 & 0.040\end{array}$

No $\quad \begin{array}{lllllll}3 & 14.3 & 18 & 85.7 & 21 & 100.0\end{array}$

Alimentación saludable

$\mathrm{Si}$

$\begin{array}{lllllll}13 & 65.0 & 7 & 35.0 & 20 & 100.0 & 0.001\end{array}$

$\begin{array}{llllll}20 & 25.5 & 60 & 75.0 & 80 & 100.0\end{array}$ 
Los adolescentes tempranos (12-14 años), tienen menor posibilidad de riesgo cardiovascular, OR 0.31 IC 95\% [0.31 - 0.76], lo que se constituye en un factor protector frente al riesgo. Los adolescentes (15-18 años) tienen 4 veces mayor riesgo cardiovascular que aquellos en las otras etapas de la adolescencia, OR: 4,36 IC 95\% [1.59 - 11.94]. El género femenino tiene 2.8 veces mayor posibilidad de riesgo cardiovascular en comparación con el género masculino, OR 2.8 IC 95\% [1.17 6.87]. Consumir bebidas alcohólicas aumenta 19.04 veces la posibilidad de riesgo cardiovascular que no consumirlas, OR 19.04 IC 95\% [2.45 - 148.11].

Aunque la inactividad física incrementa el riesgo cardiovascular, OR 3.7 IC 95\% [0.99 - 13.53], el IC pasa por el valor nulo, lo cual estadísticamente no es significativo, pero clínicamente es relevante.

No tener buenos hábitos alimenticios aumenta 5.57 veces más la posibilidad de riesgo cardiovascular, OR 5.57 IC 95\% [1.95 - 15.90]. Tabla 3.

Tabla 3. Asociación del riesgo cardiovascular con las variables significantes estadísticamente.

\begin{tabular}{lcc}
\hline Variable & OR & IC 95\% \\
\hline Variables Sociodemográficas & & \\
\hline Edad & & \\
Pre adolescentes & 1.0 & \\
Adolescentes tempranos & 0.31 & $0.13-0.76$ \\
Adolescentes & 4.36 & $1.59-11.94$ \\
Género & & \\
Femenino & 2.8 & $1.17-6.87$ \\
Masculino & 0.35 & $0.14-0.85$ \\
\hline Hábitos de vida & & \\
Consumo de bebidas alcohólicas & 19.04 & $2.45-48.11$ \\
Practica actividad física & 3.7 & $0.99-13.53$ \\
Alimentación saludable & 5.57 & $1.95-15.90$ \\
\hline
\end{tabular}

\section{Discusión}

En el estudio se encontró que la población perteneciente al género femenino, presenta más posibilidades de tener riesgo cardiovascular, lo que concuerda con lo mencionado por Claver García, la enfermedad cardiovascular siempre ha sido considerada una enfermedad de hombres, pero datos recientes de Estados Unidos demuestran que el número absoluto de mujeres que fallecen por ECV ya es mayor que el número de muertes por esta causa en los varones ${ }^{15,16}$.

Por su parte Martínez Gómez, et al. en su estudio también mostraron que las mujeres tenían niveles de adiposidad general mayores que los varones, mientras que éstos presentaban mayor nivel de obesidad abdominal ${ }^{17}$.
Con respecto a la edad, en el actual estudio se encontró un mayor riesgo cardiovascular en los adolescentes (15-18 años) con un $84 \%$, lo que concuerda con lo propuesto por Lavielle, et al. ${ }^{18}$ y es similar a los resultados de Tercedor et al. ${ }^{19}$. Los adolescentes son un grupo de riesgo debido a que han aumentado de manera importante la inactividad física y las conductas sedentarias, como resultado del uso indiscriminado de nuevas tecnologías, particularmente la televisión, la Internet, entre otros.

Respecto a los valores de presión arterial (P.A.) en este estudio se encontró que solo el 5\% tuvo presión normal alta, en concordancia con Gorrita Pérez, et al. ${ }^{20}$, en donde el $2,7 \%$ del total de adolescentes presento un valor elevado.

Según el diagnóstico nutricional en este estudio se observó que solo el $2 \%$ de la población estudiada tenían obesidad, lo cual concuerda con los resultados de Gamboa, López, et al. ${ }^{21}$. En cuanto a la glicemia en ayunas se encontró que solo el $1 \%$ presentaba cifras alteradas, resultados similares a los encontrados por Sáez, et al. ${ }^{22}$.

En el estudio, llama la atención que la prevalencia de conductas sedentarias es baja (21\%), lo anterior que puede ser debido a que la mayoría de participantes del estudio pertenecen a la zona rural (59\%), comparado con el estudio de Lavielle, et al. donde se encontró una prevalencia de inactividad física del $66,3 \%$, pero su población de estudio era urbana. Para agregar como lo señala el último estudio mencionado, una gran proporción de jóvenes en países desarrollados y en vías de desarrollo (población urbana) ven más de cuatro horas por día TV, el doble de tiempo máximo recomendado, lo que se ve reflejado en la disminución o falta de actividad física de esta población.

Respecto a la actividad física y el género se encontró que del $79 \%$ del total de adolescentes que practican actividad física, el $61 \%$ corresponde al género masculino, los resultados son similares a lo encontrado en el estudio mencionado anteriormente ${ }^{19}$, donde se evidenció que la falta de actividad física estuvo asociada con el género; en este estudio una mayor proporción de mujeres reportó ser inactiva respecto a los hombres $(75,3 \%$ vs. $54,6 \%$ respectivamente, $\mathrm{p}=0.000$ ).

Con relación a los antecedentes médicos familiares, el $71 \%$ de la población sujeto, expresaron no tener antecedentes familiares relacionados con patologías cardiovasculares diferente a los resultados de Alayón, 
et al. y quienes en su estudio encontraron que el $24,9 \%$ de los niños entrevistados expresaron no presentar antecedentes de este tipo. ${ }^{23}$

Con respecto al consumo de bebidas alcohólicas, se encontró que el $26 \%$ de la población estudiada las consume, de este porcentaje el $61.5 \%$ pertenece al género femenino, diferente a lo encontrado por Mulassi, et al. ${ }^{24} \mathrm{y}$ a los resultados obtenidos por Cando, et al. ${ }^{25}$, donde el mayor predominio de consumo fue en el género masculino.

El $6 \%$ de la población perteneciente al grupo de adolescentes fuma ocasionalmente, mientras que en el estudio de Cúneo, et al. se evidenció que el 20\% de la población consumía tabaco con regularidad ${ }^{26}$. Esta exposición de manera temprana incrementa el riesgo de desarrollar enfermedades cardiovasculares aproximadamente en un $60 \%$ en la adultez ${ }^{27}$.

En el estudio el 20\% de la población tiene buenos hábitos alimenticios, este resultado no concuerda con lo encontrado por Gorrita Pérez, et al. ${ }^{19}$, donde solo el $5.4 \%$ tenían una dieta saludable. Sin embargo, frente al consumo de comida chatarra, se encontró que el $28 \%$ no la consumen, lo que difiere con el estudio de Medrano Donlucas, et al. ${ }^{28}$. Estos resultados coinciden con la información obtenida en la Encuesta Nacional de Situación Nutrición (ENSIN) 2010, que demuestra una alimentación poco saludable en la población colombiana con un alto consumo de alimentos procesados, harinas, embutidos y con bajo consumo de frutas y verduras ${ }^{29}$.

Estos hallazgos nos sugieren que es importante reforzar en nuestra población una cultura de hábitos saludables, caracterizada por una alimentación saludable y una mayor actividad física, que constituyen dos de las principales alternativas para la prevención de enfermedades crónicas no trasmisibles ${ }^{30}$. Rodríguez Domínguez, et al. también en su estudio refieren que una vez identificados los factores de riesgo en edades tempranas se demuestra que es necesario realizar estudios de intervención inmediatos, relacionados con estilos de vida para prevenir así la enfermedad cardiovascular y la diabetes mellitus tipo 2 en la adultez ${ }^{31}$.

\section{Conclusiones}

En el estudio se encontró que dos terceras partes de la población sujeto tienen riesgo cardiovascular debido a la presencia de factores de riesgo modificables, en primer lugar los malos hábitos alimenticios relacionados con el consumo de "comida chatarra" y consumo de grasas saturadas, seguido de la falta de actividad física regular. Los adolescentes (15-18 años) tienen un riesgo cardiovascular mayor comparado con los preadolescentes y adolescentes tempranos, teniendo en cuenta que a mayor edad se incrementa de manera importante la inactividad física y las conductas sedentarias, como resultado del uso indiscriminado de nuevas tecnologías.

Se obtuvo significancia estadística entre las variables: edad, género, consumo de bebidas alcohólicas, práctica de actividad física, alimentación saludable y el riesgo cardiovascular.

El género femenino se asocia con un mayor riesgo cardiovascular a diferencia de la población masculina. Asimismo el consumo de bebidas alcohólicas, la inactividad física y los hábitos alimenticos no saludables se constituyen factores de riesgo para enfermedad cardiovascular $(\mathrm{OR}>1)$.

Por último, es fundamental involucrar de manera activa a los padres de familia, por lo cual, en la reunión donde se socializó los resultados del estudio a los padres de familia se hizo la recomendación a los tutores de la importancia que ellos tienen en la promoción de buenos hábitos alimenticios puesto que son los actores más importantes en la formación integral de sus hijos.

\section{Recomendaciones}

Debido a que la población estudiada es reducida sugerimos que se realice una investigación en una población más grande, que incluya más adolescentes de la misma institución y/o de otras instituciones educativas públicas y privadas.

La realización de nuevas investigaciones donde se evalúen poblaciones jóvenes y se identifiquen factores de riesgo en dichos grupos, generara intervenciones más efectivas principalmente desde nuestra profesión, para evitar el desarrollo de enfermedades cardiovasculares. Se propone también medir otras variables como el nivel de colesterol, nivel de estrés, etc.

El resultado de la investigación arrojó que existe riesgo cardiovascular en un gran porcentaje de la población adolescente, por lo que es necesario implementar estrategias que incluyan la práctica de actividad física regular en los estudiantes, la promoción de una alimentación saludable tanto en los colegios como en los hogares, para contribuir en la reducción de 
enfermedades cardiovasculares en edades adultas, puesto que desde la niñez se inicia la adopción de hábitos poco saludables que repercuten negativamente en edades posteriores.

\section{Agradecimientos}

Agradecemos en primera instancia a la institución educativa Concentración Escuela Guillermo Valencia, a los estudiantes que participaron en esta investigación y que proporcionaron todas las facilidades para la realización de este estudio.

A nuestra Alma Mater la Universidad del Cauca y al programa de Enfermería por brindarnos una formación integral y humana con espíritu investigativo.

A nuestros asesores: Enf. Esp. Clara Imelda Torres, Md. Mg. Diego Illera y a los demás profesores que han intervenido y colaborado de una u otra manera y a todas las personas que ayudaron significativamente en la realización de este trabajo.

\section{Conflicto de interés}

No existe conflicto de interés.

\section{Referencias}

1. Sánchez Contreras M, Moreno Gómez GA, Marín Grisales EM, García Ortiz LH. Factores de Riesgo Cardiovascular en Poblaciones Jóvenes. Rev Salud Pública. 2009; 11(1): 110-122. DOI: 10.1590/ S0124-00642009000100012.

2. OMS: Organización Mundial de la Salud. Enfermedades cardiovasculares.

3. Briceño G, Fernández M, Céspedes J. Prevalencia elevada de factores de riesgo cardiovascular en una población pediátrica. Rev Biomédica 2015; 35: 219 226. DOI: 10.7705/biomedica.v35i2.2314.

4. MINSALUD: Ministerio de salud y protección social. Enfermedades cardiovasculares.

5. OPS: Organización Panamericana de la Salud. Las dimensiones económicas de las enfermedades no transmisibles en América Latina y el Caribe.

6. Robles Valdés C. Riesgo cardiovascular y síndrome metabólico en niños y adolescentes. Rev Acta Pediatr Mex. 2011; 32(1): 1-4.

7. López Cortés LF, Cifuentes Ortiz M, Sánchez Ruiz A. Calidad de vida relacionada con la salud en pacientes con diabetes mellitus tipo 2, en un hospital de mediana complejidad en Cali. Ciencia y salud, Universidad Santiago de Cali. 2014; 2(8): 43-48.
8. Instituto Nacional de Salud. Las enfermedades cardiovasculares: un problema de salud pública y un reto global. Rev Biomédica. 2011; 31(4).

9. Instituto Nacional de Salud. Boletín del observatorio en salud.

10.Zea Robles AC, Leon Ariza HH, Botero Rosas DA, Afanador Castañeda HD, Pinzón Bravo LA. Factores de riesgo cardiovascular y su relación con la composición corporal en estudiantes universitarios. Rev Salud Pública. 2014; 16(4): 505-515. DOI: 10.15446/rsap

11. Campos Cavada I. Factores de riesgo modificables para enfermedad cardiovascular en niños. Rev An Venez Nutr. 2010; 23(2): 100-107.

12.Texas Heart Institute. Factores de riesgo cardiovascular para niños y adolescentes.

13.Juonala M, Magnussen CG, Berenson GS, Venn A, Burns TL, Sabin MA, et al. Childhood Adiposity, Adult Adiposity, and Cardiovascular Risk Factors. New Engl J Med. 2012; 67(3): 156-158. DOI: 10.1097/OGX.0b013e318248378.

14.García Gulfo MA, García Zea JA. Prevalencia de factores de riesgo cardiovascular en jóvenes de una institución universitaria. Rev Salud Pública. 2012; 14(5): 822-830. DOI: 10.15446/rsap.

15. Claver García, Laura. Riesgo Cardiovascular en la mujer. Universidad Pública de Navarra. 2014.

16.Alfonso F, Bermejo J, Segovia J. Enfermedades cardiovasculares en la mujer: ¿por qué ahora? Rev Esp Cardiol. 2006; 59(3): 259-263. DOI: $10.1157 / 13086084$.

17.Martínez Gómez D, Eisenmann J, Gómez Martínez $\mathrm{S}$, Vesesa A, Ascensión M, Veiga O. Sedentarismo, adiposidad y factores de riesgo cardiovascular en adolescentes. Estudio AFINOS. Rev Esp Cardiol. 2010; 63(3): 277-285. DOI: 10.1157/13086084.

18.Lavielle Sotomayor P, Pineda Aquino V, Jáuregui Jiménez O, Castillo Trejo M. Actividad física y sedentarismo: determinantes sociodemográficos, familiares y su impacto en la salud del adolescente. Rev Salud pública 2014; 16(2): 161-172. DOI: 10.15446/rsap.

19.Tercedor P, Matillas M, Chillón P, Pérez López IJ, Ortega FB, Wärnberg $J$, et al. Incremento del consumo de tabaco y disminución del nivel de práctica de actividad física en adolescentes españoles: Estudio AVENA. Nutr Hosp. 2007; 22(1): 89-94.

20.Gorrita Pérez R, Ruiz King Y, Hernández Martínez Y, Sánchez Lastre M. Factores de riesgo de enfermedades cardiovasculares y cerebrovasculares en adolescentes. Rev Cub Pediat. 2015; 87(2): 140-155. 
21.Gamboa Delgado EM, López Barbosa N, Quintero Lesmes DC. Prevalencia de sobrepeso y obesidad en adolescentes del municipio de Floridablanca, Colombia. Rev Med UNAB, 2007; 10(2): 1-8.

22.Sáez Y, Bernui I. Prevalencia de factores de riesgo cardiovascular en adolescentes de instituciones educativas. Ann Fac Med. 2009; 70(4): 259-265. DOI: 10.15381/anales.v70i4.926.

23.Alayón A, Castro Orozco R, Gaviria Esquivia L, Fernández Franco M, Benítez Peña L. Factores de riesgo cardiovascular en escolares entre 7 y 14 años en Cartagena, Colombia, 2009. Rev Salud Pública. 2011; 13(2): 196-206. DOI: 10.15446/rsap.

24.Mulassi A, Hadid C, Borracci R. Hábitos de alimentación, actividad física, tabaquismo $\mathrm{y}$ consumo de alcohol en adolescente escolarizado de la provincia y el conurbano bonaerenses. Arch Argent Pediatr. 2010; 108(1): 45-54.

25.Cando J, Chuya V. Factores de riesgo del consumo de alcohol y su influencia en el rendimiento académico de adolescentes del colegio "Miguel Malo González” del cantón Gualaceo, Azuay 2016. Repositorio Institucional Uni Cuenca, Ecuador.

26. Cuneo F, Contini MC, Zino GD. Factores de riesgo cardiovascular y sus asociaciones en adolescentes de la ciudad de Santo Tomé. Rev FABICIB. 2015; 31(142): 34-41.

27.Lanas F, Serón, P. Rol del tabaquismo en el riesgo cardiovascular global. Rev Med Clin Condes. 2012; 23(6): 699-705.

28.Donlucas G, Medrano JA, Acosta Favela ME, Ruiz Morales JA, Vargas E. Principales Factores de riesgo cardiovascular en escolares. CULCyT. 2014; 54(1): 72-80.

29.MinSalud: Ministerio de salud y protección social. Encuesta Nacional de Situación Nutrición (ENSIN) 2010.

30.Obregón MC, Celis LG, De Jesús Ayala J, Domínguez T, Girata MV, Almonacid CC. Factores promotores de cultura nutricional en adolescentes y adultos jóvenes de la Universidad de la Sabana. Rev Cient Salud Uninorte. 2013; 29(3): 374-383.

31.Rodríguez Domínguez L, Díaz Sánchez ME, Ruiz Álvarez V, Hernández Hernández $\mathrm{H}$, Herrera Gómez V, Montero Díaz V. Factores de riesgo cardiovascular y su relación con la hipertensión arterial en adolescentes. Rev Cub Med. 2014; 53(1): 25-36. 